\title{
Reversible splenial lesion syndrome associated with acute Mycoplasma pneumoniae-associated encephalitis: A report of four cases and literature review
}

\author{
XIAOYU DONG and SHUYAN CONG \\ Department of Neurology, Shengjing Hospital of China Medical University, Shenyang, Liaoning 110004, P.R. China
}

Received October 18, 2017; Accepted June 8, 2018

DOI: $10.3892 /$ etm.2018.6387

\begin{abstract}
Reversible splenial lesion syndrome (RESLES) is a rare clinico-radiological syndrome that is defined as reversible lesions that involve the splenium of the corpus callosum (SCC). RESLES has been reported in patients with a broad spectrum of diseases and conditions, including infections, hypoglycemia and poisoning. The present report described four RESLES cases triggered by Mycoplasma pneumoniae (M.pneumoniae) and discussed the associated diagnostic challenges. Four cases of acute M.pneumoniae-associated encephalitis that displayed RESLES were reviewed. The clinical presentations were nonspecific in these patients. However, magnetic resonance imaging (MRI) revealed consistent lesions in the SCC with a hyperintensity in diffusion-weighted imaging (DWI) and hypointensities in T1WI, which disappeared after a variable lapse. Treatment with azithromycin or combined treatment with immunomodulatory agents if necessary led to a good prognosis. The present findings indicated that radiological diagnosis of RESLES should be considered in patients with M.pneumoniaeassociated encephalitis. Furthermore, serum Mycoplasma antibody is important for the diagnosis of M. pneumoniae-associated encephalitis.
\end{abstract}

\section{Introduction}

Mycoplasma pneumoniae (M. pneumoniae) is a common respiratory tract pathogen, especially in children (1-3). Up to $40 \%$ of cases of community-acquired pneumoniae admitted to the hospital are due to $M$. pneumoniae infection. The central nervous system (CNS) may be one of the most susceptible sites of M. pneumoniae besides the respiratory system (4). M. pneumoniae infection accounts for

Correspondence to: Dr Shuyan Cong, Department of Neurology, Shengjing Hospital of China Medical University, 36 Sanhao Street, Heping, Shenyang, Liaoning 110004, P.R. China

E-mail: congsy_cmu@outlook.com

Key words: Mycoplasma pneumoniae-associated encephalitis, reversible splenial lesion syndrome, magnetic resonance imaging, serum antibody
$5-10 \%$ of pediatric encephalitis patients and up to $60 \%$ of these patients show neurologic sequelae and neuroimaging discloses normal findings or focal diffuse edema (5). There have been few reports of the clinical characteristics of adult M. pneumoniae-associated encephalitis and the use of magnetic resonance imaging (MRI) in the diagnosis.

Reversible splenial lesion syndrome (RESLES) is a rare clinico-radiological syndrome that is defined as reversible lesions that involve the splenium of the corpus callosum (SCC). RESLES has been reported in patients with a broad spectrum of diseases and conditions, including infections, hypoglycemia, and poisoning. However, the pathophysiological mechanism of RESLES is not well known (6).

We report four cases of adult mycoplasma-associated encephalitis in which MRI scan revealed reversible lesions in RESLES. Complete recovery of clinical symptoms and signs were observed in a few days or weeks after treatment.

Is a common respiratory tract pathogen, especially in children (1-3). Up to $40 \%$ of cases of community-acquired pneumoniae admitted to the hospital are due to M.pneumoniae infection. The CNS may be one of the most susceptible sites of M. pneumoniae besides the respiratory system (4). M. pneumoniae infection accounts for $5-10 \%$ of pediatric encephalitis patients and up to $60 \%$ of these patients show neurologic sequelae and neuroimaging discloses normal findings or focal diffuse edema (5). There have been few reports of the clinical characteristics of adult $M$. pneumoniae-associated encephalitis and the use of MRI in the diagnosis.

RESLES is a rare clinico-radiological syndrome that is defined as reversible lesions that involve the SCC. RESLES has been reported in patients with a broad spectrum of diseases and conditions, including infections, hypoglycemia, and poisoning. However, the pathophysiological mechanism of RESLES is not well known (6).

We report four cases of adult mycoplasma-associated encephalitis in which MRI scan revealed reversible lesions in RESLES. Complete recovery of clinical symptoms and signs were observed in a few days or weeks after treatment.

\section{Case report}

Between March 2011 and November 2016, four patients who presented with fever and headache were admitted to Shengjing Hospital. Examinations of blood and cerebrospinal fluid (CSF), 
and MRI scans were completed in the days after admission. The two male and two female patients in this retrospective study had an average age of $29.5 \pm 11.0$ years. None of the patients had suffered from brain trauma, toxic or metabolic encephalitis/encephalopathy, cerebral vascular diseases, and cerebral neoplasma.

MRI was performed using 3.0 T units that comprised axes of T1-and T2-weighted sequences. Fast fluid attenuated inversion recovery (FLAIR) sequences and diffusion-weighted imaging (DWI) were performed using an axial multi-section single-shot echo-planar SE sequence. Enhanced T1-weighted scans obtained after intravenous administration of $10 \mathrm{ml}$ of gadoteric acid $0.5 \mathrm{mmol} / \mathrm{ml}$. MRI findings, such as the shape of the splenial lesion, and the DWI and FLAIR findings were evaluated by two neuroimaging specialists with over 10 years experience each. For M. pneumoniae serology detection, IgM and IgG antibodies were detected using the SeroMP IgM and IgG kit (Tecan Group, Ltd., Mannedorf, Switzerland), which is a semi-quantitative ELISA to determine the antibodies specific to M. pneumoniae. The antigen used in the SeroMP kit is the P1 membrane protein, which is the most important virulence factor of $M$. pneumoniae (7). Based on the manufacturer's suggestion, a positive $\operatorname{IgM}$ as $\geq 1.1 \mathrm{~S} / \mathrm{CO}$, the quantitative $\mathrm{IgG}$ was also detected between 7 to 14 days after the first test, while recording the multiplier of the increase of $\operatorname{IgG}$ ( $\mathrm{IgG} \geq 22 \mathrm{RU} / \mathrm{ml}$ as positive). An IgM positive reaction together with an increase of $\mathrm{IgG}$ exceeding 4 times in patients indicated the presence of $M$. pneumoniae acute infection (8). Other laboratory tests including biochemical and microbiological examinations of the CSF were available in all cases. After treatment with azithromycin, the clinical symptoms and signs recovered quickly. A follow-up MRI showed all lesions in SCC were reversible at 10, 24, 12, and 7 days in case 1, 2, 3, and 4 , respectively.

\section{Description of cases}

Case 1. A 31-year-old Chinese man was transferred to our emergency room from a nearby primary hospital. He had developed a cold and a fever 3 days previously. His body temperature reached $39^{\circ} \mathrm{C}$. He had persistent headaches. After 2 days' treatment in the primary hospital, the fever and headache were not improved. At admission, we found he had mild somnolence and Kernig sign (+), with other neurological examinations being negative. Chest computed tomography (CT) revealed an infective lesion in the right lung lobe. In the third day following administration, a cranial MRI scan showed focal hyper-intensities signals on the DWI, T2, and FLAIR sequences and hypo-intensities on the T1 sequence in SCC (Fig. 1). Routine blood laboratory examination revealed no obvious abnormalities. Serum M. pneumoniae antibody showed IgM positive, with an IgG titer elevated to $1: 80$. Serologic and pathogenic detection for other pathogens including Chlamydia pneumoniae, varicella zoster virus (VZV), mumps virus, measles virus, herpes simplex virus (HSV) 1 and 2, cytomegalovirus (CMV) and Japanese encephalitis virus (JEV) were negative. CSF analysis showed a high pressure $\left(200 \mathrm{mmH}_{2} \mathrm{O}\right)$, a normal total white cell count of $6 \times 10^{6} / 1$ (reference range $0-5 \times 10^{6} / 1$ ), CSF protein of $0.25 \mathrm{~g} / 1$ (reference range $0.15-0.45 \mathrm{~g} / \mathrm{l}$ ), glucose of $4.14 \mathrm{mmol} / 1$ (reference range 2.5-4.5 mmol), and chloride of $120.6 \mathrm{mmol} / \mathrm{l}$ (reference range 120-132 mmol/l). General bacteria, tubercle bacilli, and cryptococcus were not detected. Considering the clinical and serological results together with the radiological findings, acute encephalitis due to $M$. pneumoniae infection was diagnosed. Patient was treated with intravenous azithromycin $(10 \mathrm{mg} / \mathrm{kg} / \mathrm{day})$ for 2 weeks. The body temperature returned to normal 3 days later during hospitalization. The headache improved significantly after 6 days. Cranial MRI scan performed at day 10 following admission showed the complete disappearance of focal hyperintersities in SCC (Fig. 2). The serum M. pneumoniae antibody IgG was retested at day 12 ; an elevated titer of 1:1,280 was evident. The results confirmed the diagnosis of M.pneumoniae encephalitis.

Case 2. A week prior to admission, a 33-year-old Chinese woman developed headache and fever without obvious predisposing causes. The highest body temperature was $37.8^{\circ} \mathrm{C}$. Four days later, the patient developed blurred vision and a paroxysmal amaurosis. A brain CT at that time performed at the fourth hospital affiliated to China medical university revealed no abnormalities. Specific serum $M$. pneumoniae antibody was markedly elevated to 1:320 on day 6 after onset. CSF analysis was abnormal with a pressure of $290 \mathrm{mmH}_{2} \mathrm{O}, 316 \times 10^{6} / 1$ white cells, and $1.32 \mathrm{~g} / 1$ protein. Other laboratory tests of CFS, including general bacteria, tubercle bacilli, and cryptococcus were all negative. At that time, the patient was treated with mannitol (250 $\mathrm{ml}$ every $8 \mathrm{~h})$, ganciclovir $(50 \mathrm{mg} / \mathrm{kg} /$ day with treatment every $12 \mathrm{~h})$ and azithromycin $(10 \mathrm{mg} / \mathrm{kg} / \mathrm{day})$. Symptoms did not improve. The patient displayed nausea and vomiting, and body temperature reached $39.5^{\circ} \mathrm{C}$. The patient was transferred to our hospital on day 9. The patient had no past history of other related diseases except gastric ulcer and hypertension. Neurological examination on administration showed poor mental status and drowsiness, limb muscle strength grade 4 , and neck rigidity (+). Other neurological signs were negative. Laboratory examination showed specific serum $M$. pneumoniae antibody (IgM) was positive and IgG antibody titer was elevated to 1:640. Serology for other pathogens including $C$. pneumoniae, VZV, mumps virus, measles virus, HSV 1 and 2, CMV, and JEV were negative. $\mathrm{CSF}$ analysis showed a pressure of $350 \mathrm{mmH}_{2} \mathrm{O}$, Pandy reaction (+), an abnormal white cell count of $286 \times 10^{6} / 1$, monocyte percentage $96.9 \%$, protein $0.96 \mathrm{~g} / 1,2.46 \mathrm{mmol} / \mathrm{l}$ glucose, and $119.7 \mathrm{mmol} / \mathrm{l}$ chloride. Chest CT scan showed infective lesions in the middle lobe of the right lung and the upper lobe of the left lung. Brain enhanced MRI showed focal high-signal lesion in the SCC on DWI, indicating a cytotoxic edema (Fig. 3). The clinical and serological results together with the radiological findings lead to a diagnosis of acute encephalitis due to $M$. pneumoniae infection and intrapulmonary infection. Azithromycin $(10 \mathrm{mg} / \mathrm{kg} /$ day $)$ was administered for 10 days. Mannitol (250 $\mathrm{ml}$ every $8 \mathrm{~h}$ ) was given to reduce intracranial pressure. Methylprednisolone (20 mg/kg/day) for a week, and immunoglobulin $(0.4 \mathrm{~g} / \mathrm{kg} / \mathrm{day})$ were also given for 5 days for immunoregulation and to inhibit the inflammatory response. The symptoms improved significantly. Ten days after admission, the patient no longer had headache and fever. Neurological examination showed fully-recovered right limb muscle strength and neck rigidity (-). Serum M. pneumoniae antibody IgG titer was markedly elevated to $1: 1280$. CSF 

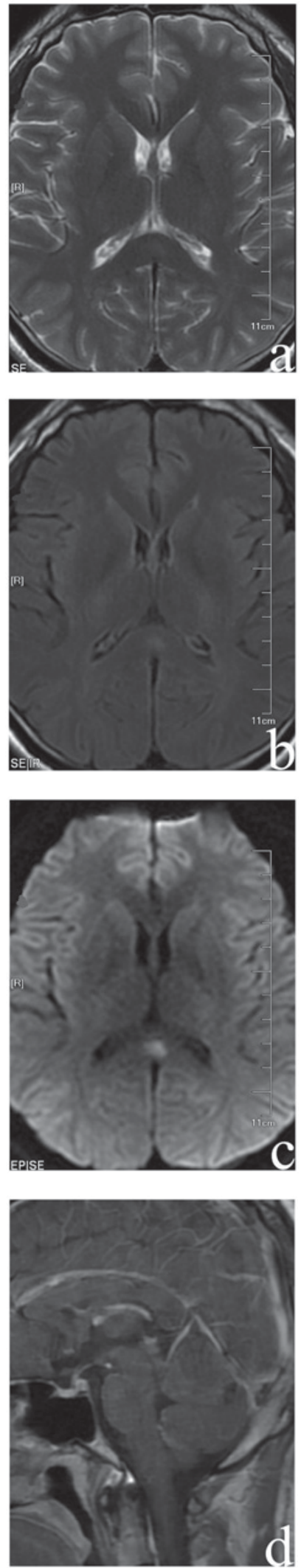

Figure 1. Patient 1 MRI: (A) T2 W, (B) FLAIR, (C) DWI sequences showed hyperintensity signal in the splenium of corpus callosum, (D) sagittal T1 W enhanced scanning showed no enhancement effect in the lesion. The same region displayed hypointensity. MRI, magnetic resonance imaging; FLAIR, fluid attenuated inversion recovery; DWI, diffusion-weighted imaging.

analysis showed a pressure of $200 \mathrm{mmH}_{2} \mathrm{O}$, Pandy reaction (-), an abnormal white cell count $\left(86 \times 10^{6} / 1\right)$, monocyte percentage
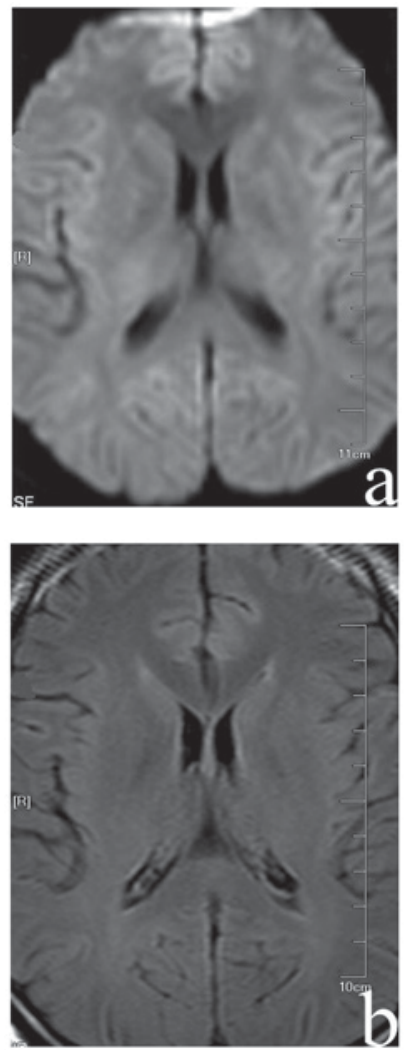

Figure 2. Patient 1 follow-up MRI 10 days after admission. (A) DWI and (B) FLAIR sequences showed focal high-signal lesion in the splenial disappeared completely. MRI, magnetic resonance imaging; DWI, diffusion-weighted imaging; FLAIR, fluid attenuated inversion recovery.

of $96.5 \%$, protein $0.69 \mathrm{~g} / \mathrm{l}$, glucose $2.91 \mathrm{mmol} / \mathrm{l}$, and chloride $121.1 \mathrm{mmol} / \mathrm{l}$. A follow-up brain MRI on day 24 of the disease revealed no abnormalities (Fig. 4).

Case 3. A 40-year-old man was admitted to the hospital from a nearby primary hospital with headache and fever for more than 10 days that occurred after overworking and becoming chilled. The highest body temperature of the patient was $38.9^{\circ} \mathrm{C}$. The patient went to a nearby hospital when his vision blurred. At that time, mannitol, ganciclovir, and ceftriaxone were given for treatment (the detailed dosages are unknown). The headache did not improve and he became disoriented. The patient was transferred to our hospital. He had no past history of other related disease, except acute hepatitis A and chronic cholecystitis. Neurological examination on admission revealed drowsiness, limb muscle strength grade 4+, Kernig sign (+), and neck rigidity (+). Other neurological examinations were negative. Cranial MRI scan done on 2015-5-14 at the local hospital revealed no obviously abnormalities. Chest CT scan done at Shengjing Hospital on 2015-05-20 revealed double infective lesions. Brain enhanced MR scan done at Shengjing Hospital on 2015-05-22 showed focal signal hyper-intensities on the T2, DWI, FLAIR sequence, and hypo-intensities on the T1 sequence in SCC (Fig. 5). Laboratory examination revealed markedly elevated serum $M$. pneumoniae antibody titer $(1: 1,280)$. Serology for other pathogens including C. pneumoniae, VZV, mumps virus, measles virus, HSV 1 and 2, CMV, and JEV were negative. CSF analysis revealed a pressure of $310 \mathrm{mmH}_{2} \mathrm{O}$, Pandy reaction (++), an abnormal white 

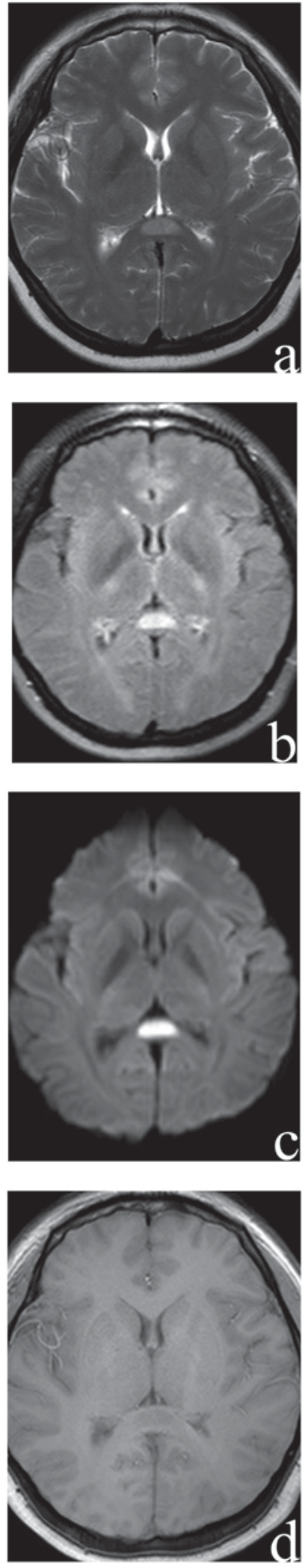

Figure 3. Patient 2 MRI: (A) T2W, (B) FLAIR, (C) DWI sequences showed hyperintensity signal in the splenium of corpus callosum, and (D) T1 W showed the same region of hypointensity. MRI, magnetic resonance imaging; FLAIR, fluid attenuated inversion recovery; DWI, diffusion-weighted imaging.

cell count $\left(86 \times 10^{6} / 1\right)$, monocyte percentage of $98.7 \%$, protein $2.83 \mathrm{~g} / 1$, glucose $2.24 \mathrm{mmol} / 1$, and chloride $122.6 \mathrm{mmol} / 1$. General bacteria, tubercle bacilli, and cryptococcus were
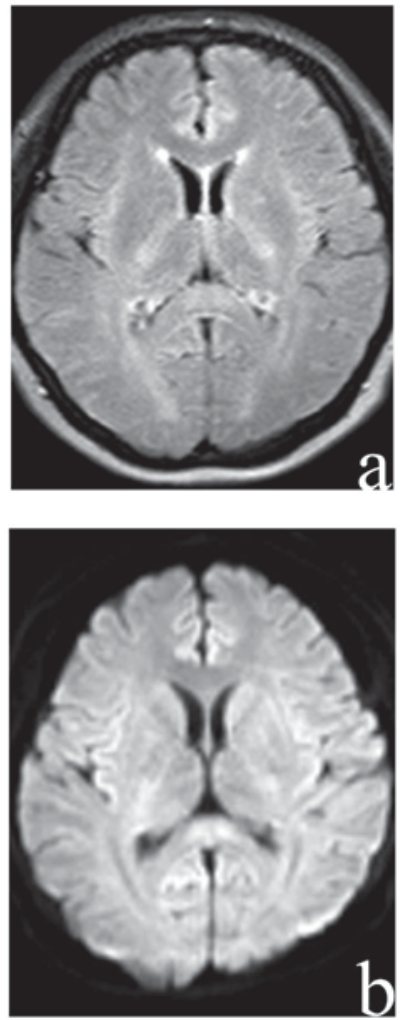

Figure 4. Patient 2 follow up MRI 15 days after admission. (A) FLAIR and (B) DWI hyperintensities signal had disappeared completely in the splenial region. MRI, magnetic resonance imaging; FLAIR, fluid attenuated inversion recovery; DWI, diffusion-weighted imaging.

not detected. The clinical and serological results (especially elevated serum $M$. pneumoniae antibody titer) together with the radiological findings supported a diagnosis of acute Mycoplasma encephalitis. Azithromycin (10 mg/kg/day) was administrated for treatment, while mannitol (250 $\mathrm{ml}$ every $8 \mathrm{~h}$ ) was given to reduce intracranial pressure. The treatment was effective. On the seventh day after admission, the headache and blurred vision had significantly improved. Fever remained intermittent and the body temperature reached $37.5^{\circ} \mathrm{C}$. On day 14 after admission, body temperature had returned to normal and $M$. pneumoniae antibody titer determined on 2015-05-31 had markedly declined to 1:160. A follow-up brain MRI scan on 2015-06-02 did not show obvious anomalies (Fig. 6).

Case 4. A 14-year-old female patient was transferred to our hospital due to fever and headache for one week, accompanied with vomiting twice. Physical examination showed a body temperature of $38.8^{\circ} \mathrm{C}$. Neurological examination found neck rigidity (+). Other neurological examinations were normal. Chest CT scan done on 2016-11-21 revealed some inflammation in the upper segment of the inferior lobe of the right lung and frontier segment of the superior lobe of the left lung. Cranial MRI done on 2016-11-22 showed focal hyper-intensities signal on the T2, DWI, FLAIR sequence, and hypo-intensities on the T1 sequence in SCC (Fig. 7). Laboratory examination showed $M$. pneumoniae $\operatorname{IgM}$ antibody positive, and the $\operatorname{IgG}$ titer was 1:160. Serology for other pathogens including C. pneumoniae, VZV, mumps virus, measles virus, HSV 1 and 2, CMV and JEV were negative. CSF analysis showed a pressure of $200 \mathrm{mmH}_{2} \mathrm{O}$, an 

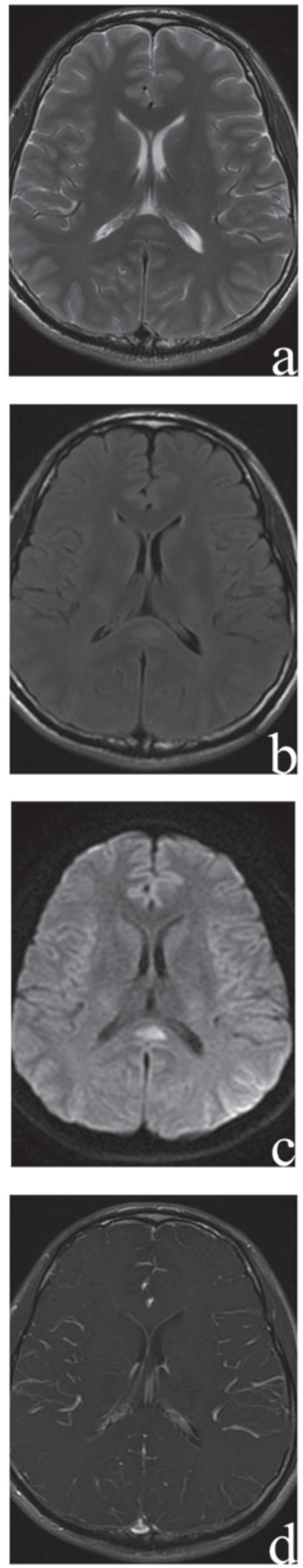

Figure 5. Patient 3 MRI: (A) T2W, (B) FLAIR, (C) DWI sequences showed hyperintensity signal in the splenium of corpus callosum and (D) T1W enhanced scanning showed no enhancement effect. MRI, magnetic resonance imaging; FLAIR, fluid attenuated inversion recovery; DWI, diffusion-weighted imaging.

abnormal white cell count (341x10\%1), 96.7\% monocytes, Pandy reaction (+), protein $1.42 \mathrm{~g} / \mathrm{l}$, with normal glucose and chloride.
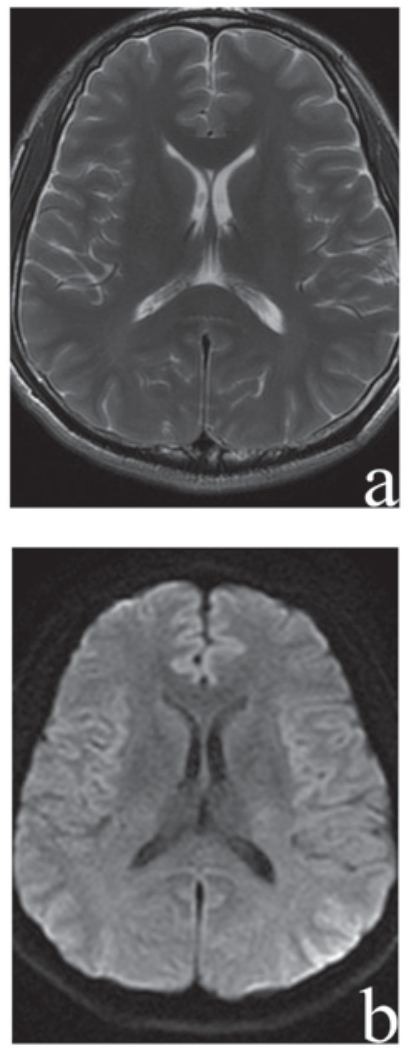

Figure 6. Patient 3 follow up MRI 12 days after admission. (A) T2 and (B) DWI hyperintensities signal had disappeared completely in the splenial region. MRI, magnetic resonance imaging; DWI, diffusion-weighted imaging.

General bacteria, tubercle bacilli, and cryptococcus were not detected. The combined clinical and serological results together with the radiological findings supported a diagnosed as acute Mycoplasma encephalitis. The patient was treated with intravenous azithromycin $(10 \mathrm{mg} / \mathrm{kg} / \mathrm{day})$ for 2 weeks. The symptoms improved significantly at the sixth day after admission. At that time the headache and vomiting had ended, and she had normal body temperature. Two weeks after treatment, neurological examination showed Kernig sign (-), neck rigidity (-), and other neurological examinations were all negative. Re-examination of the cranial MRI on 2016-11-28 showed that the high signal of SCC had decreased (Fig. 8). Re-examination of CSF analysis on 2016-12-06 showed a pressure of $150 \mathrm{mmH}_{2} \mathrm{O}$, abnormal white cell count $\left(50 \times 10^{6} / 1\right), 98 \%$ monocytes, Pandy reaction (+), protein $0.78 \mathrm{~g} / 1$, and normal glucose and chloride.

\section{Discussion}

Adult CNS involvement associated with $M$. pneumoniae infection is being reported with increasing frequency $(9,10)$, but the diagnostic criteria, clinical manifestations, and cranial imaging changes of M. pneumoniae encephalitis are still not elucidated. In this case series, we observed four clinically confirmed M. pneumoniae encephalitis patients, all of whom had transient SCC lesions in cranial MRI. Through the review of this series of cases, we hope to help clinicians to get a better understanding of M. pneumoniae encephalitis.

Despite advances in diagnostic techniques, the diagnosis of M. pneumoniae encephalitis is still challenging. 

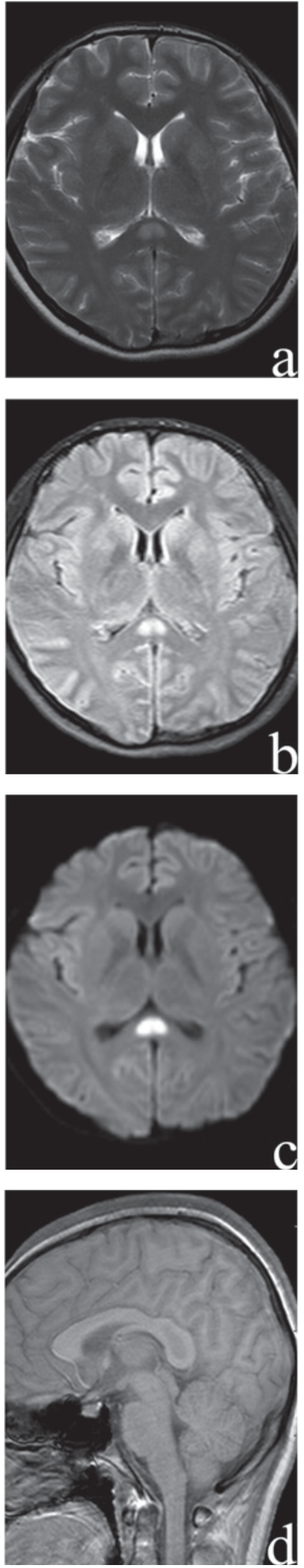

Figure 7. Patient 4 MRI: (A) T2W, (B) FLAIR, (C) DWI sequences showed hyperintensity signal in the splenium of corpus callosum and (D) sagitta T1W sequence showed the same region of hypointensity. MRI, magnetic resonance imaging; FLAIR, fluid attenuated inversion recovery; DWI, diffusion-weighted imaging.

Although depending on the serological testing for diagnosis of M. pneumoniae encephalitis may be problematic, it is
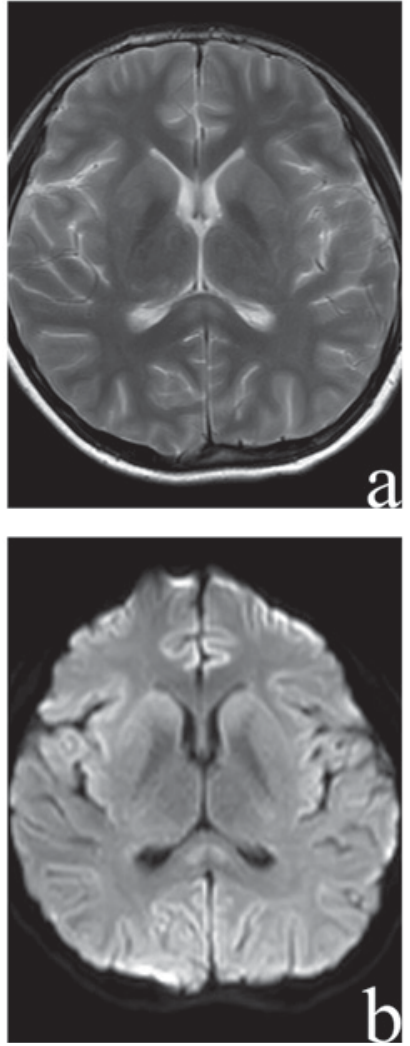

Figure 8. Patient 4 follow up MRI 7 days after admission. (A) T2 and (B) DWI hyperintensities signal had decreased obviously in the splenial region. MRI, magnetic resonance imaging; DWI, diffusion-weighted imaging.

undeniable that serological testing is the mainstay for diagnosis of $M$. pneumoniae infection, especially in developing countries where PCR technology and Western blot are not readily available. Additionally, serological test saves more time than Mycoplasma culture, and may reach a similar sensitivity as PCR for diagnosis of acute infection of M. pneumoniae $(11,12)$. In our study, one of our diagnostic staples was the detection of serum antibody. Specific IgM antibody to M.pneumoniae in an acute infectious phase responses usually in the first 7-10 days of illness and precedes IgG response by a few days (13). A four-fold increase in titer between acute and convalescent sera of $\mathrm{IgG}$ is also important to patients' diagnosis. In our cases, all four adult patients have 4-fold increase in IgG, confirming the M. pneumoniae infection (14).

CNS disorders, such as encephalitis, meningitis, cerebellitis, and myelitis, are common extrapulmonary manifestations of M. pneumoniae infection (15). One of the most common clinical manifestations of $M$. pneumoniae encephalitis in adults is encephalopathy. Symptoms may range from a subtle effect on mental status with personality and behavioral changes to stupor and coma (16). Other frequently reported manifestations include epileptic activity, meningeal signs (i.e., headache, neck stiffness, vomiting) and ataxia. Focal neurological deficits may be observed as well $(8,17,18)$. In the present study, all patients had fever and headache, three of four had neck stiffness, two had disturbance of consciousness, and one patient had visual disturbance, which was consistent with previous reports.

The MRI findings of M. pneumoniae-associated encephalitis are quite variable and haphazard. It is more sensitive than 
$\mathrm{CT}$ in the detection of subtle lesions even in the early stage of infection (19). However, the proportion of imaging abnormalities is still unclear (20). The involved regions include the cerebral cortex, hemisphere, basal ganglia, hippocampus, cerebellum, and corpus callosum (21). We observed reversible focal lesions in the SCC in all four patients, which has been reported and defined as RESLES in hypoglycemia, hypernatremia, high-altitude cerebral edema or antiepileptic drug toxicity or withdrawal (6), but has rarely been reported in acute $M$. pneumoniae-associated encephalitis. We reported the largest number of acute M.pneumoniae-associated encephalitis with RESLES to date. The pathophysiological mechanisms of M. pneumoniae-associated encephalitis, especially selective splenial lesions in the SCC, are not very clear. It has been proposed that the specific affinity of infective/auto-immunological antigens or induced antibodies to the splenial axonal receptors are responsible for the splenial involvement in infective/auto-immunological encephalitis $(22,23)$. Moreover, it has been postulated that the SCC results in specific vulnerability to excitotoxicity injury in metabolic diseases, which makes this area selectively involved in different pathological events (24). Besides these two possible mechanisms, direct infection of the brain could be another underlying mechanism for post infectious complications of M. pneumoniae infection, but it is incapable of explaining the solitary involvement of the SCC (16). Therefore, we speculate that autoimmune-mediated brain injury may be a possible reason. In this scenario, M. pneumoniae-derived antigenic elements could bound to blood macrophages or monocytes may initiate complex immune reactions (25). The CSF analyses of our cases showed the clearly increased percentage of monocytes, which might be related with immune complex formation and could further lead to subsequent neurological damage (16). Other disorders that could lead to RESLES include viral encephalitis, antiepileptic drug toxicity or withdrawal, hypoglycemia, and autoimmune encephalopathy/encephalitis (6). All of the aforementioned disorders could be excluded in the four patients described in this study. The role of antibiotic in the treatment of M. pneumoniae associated encephalitis remains unresolved because of the lack of controlled trials $(2,26)$. Several therapeutic measures including antibiotics, corticosteroids, intravenous immunoglobulin and plasmapheresis have been reported for treatment of M. pneumoniae-associated encephalitis (27-29). The macrolides are considered the first-line agents for $M$. pneumoniae infections, although such antibiotics may poorly traverse the blood-brain barrier (30). Despite lack of the available literature data that may help to provide guidance regarding the efficacy of antibiotic therapies, azithromycin was administrated to treat our patients and good curative effects were obtained. As the possible immunological origin of $M$. pneumoniae-associated encephalitis, immunomodulatory treatment may help (31). One of our patients received corticosteroids combined with intravenous immunoglobulin; good prognosis was obtained in both the respiratory and nervous systems.

In this case series, four cases with the involvement of splenium due to M. pneumoniae infection are described. All cases received azithromycin treatment alone or combined with immunomodulatory treatment. All of the patients made a full recovery and we found reversible signal changes in MRI findings in the corpus callosum lesions during the follow-up periods. Further studies should be performed to clarify the possible mechanisms underlying CNS involvement due to M. pneumoniae infection and controlled trials should be conducted to assess the effectiveness of therapeutic methods.

\section{Acknowledgements}

This work was supported by the National Natural Science Foundation of China (no. 81371271), and was also sponsored by 'Liaoning BaiQianWan Talents Program'.

\section{Funding}

The present study received funding from the National Natural Science Foundation of China (grant no. 81371271).

\section{Availability of data and materials}

The datasets used and/or analyzed during the present study are available from the corresponding author on reasonable request.

\section{Authors' contributions}

XD was responsible for collecting the cases and writing articles and SC was responsible for the design of the present study. All authors read and approved the final manuscript.

\section{Ethics approval and consent to participate}

All procedures performed in the studies involving human participants were in accordance with the ethical standards of the institutional and/or national research committee and performed following the guidelines of the 1964 Helsinki Declaration and its later amendments or comparable ethical standards.

\section{Patient consent for publication}

Consent for publication was obtained from the patients of this study.

\section{Competing interests}

The authors declare that they have no competing interests.

\section{References}

1. Lind K, Benzon MW, Jensen JS and Clyde WA Jr: A seroepidemiological study of Mycoplasma pneumoniae infections in Denmark over the 50-year period 1946-1995. Eur J Epidemiol 13: 581-586, 1997.

2. Bébéar C, Dupon M, Renaudin H and de Barbeyrac B: Potential improvements in therapeutic options for mycoplasmal respiratory infections. Clin Infect Dis 17 (Suppl 1): S202-S207, 1993.

3. Foy HM: Infections caused by Mycoplasma pneumoniae and possible carrier state in different populations of patients. Clin Infect Dis 17 (Suppl 1): S37-S46, 1993.

4. Narita M: Pathogenesis of neurologic manifestations of Mycoplasma pneumoniae infection. Pediatr Neurol 41: 159-166, 2009.

5. Kuwahara M, Samukawa M, Ikeda T, Morikawa M, Ueno R, Hamada Y and Kusunoki S: Characterization of the neurological diseases associated with Mycoplasma pneumoniae infection and anti-glycolipid antibodies. J Neurol 264: 467-475, 2017.

6. Zhang S, Ma Y and Feng J: Clinicoradiological spectrum of reversible splenial lesion syndrome (RESLES) in adults: A retrospective study of a rare entity. Medicine (Baltimore) 94: e512: 2015. 
7. Rastawicki W, Rokosz N, Gierczyński R and Jagielski M: Use of recombinant P1 protein of Mycoplasma pneumoniae for the serodiagnosis of mycoplasmosis. Med Dosw Mikrobiol 64: 229-237, 2012 (In Polish).

8. Jacobs E: Serological diagnosis of Mycoplasma pneumoniae infections: A critical review of current procedures. Clin Infect Dis 17 (Suppl 1): S79-S82, 1993.

9. Guo ZN, Zhang HL, Bai J, Wu J and Yang Y: Meningitis associated with bilateral optic papillitis following Mycoplasma pneumoniae infection. Neurol Sci 33: 355-358, 2012

10. Narita M: Acute necrotizing encephalopathy by Mycoplasma pneumoniae infection? Arch Intern Med 162: 1647, 2002.

11. Li SL, Sun HM, Zhao HQ, Cao L, Yuan Y, Feng YL and Xue GH: A single tube modified allele-specific-PCR for rapid detection of erythromycin-resistant Mycoplasma pneumoniae in Beijin. Chin Med J (Engl) 125: 2671-2676, 2012.

12. Waris ME, Toikka P, Saarinen T, Nikkari S, Meurman O, Vainionpää R, Mertsola J and Ruuskanen O: Diagnosis of Mycoplasma pneumoniae pneumonia in children. J Clin Microbiol 36: 3155-3159, 1998

13. Li S, Zhao H, Sun H, Feng Y, Xue G and Yan C: Comparison of culture method, polymerase chain reaction and serological test for the detection of Mycoplasma pneumoniae infection in children with pneumoniae. Chin J Microbiol Immunol 37: 73-77, 2017.

14. Meyer Sauteur PM, Jacobs BC, Spuesens EB, Jacobs E, Nadal D, Vink C and van Rossum AM: Antibody responses to Mycoplasma pneumoniae: Role in pathogenesis and diagnosis of encephalitis? PLoS Pathog 10: e1003983, 2014.

15. Al-Zaidy SA, MacGregor D, Mahant S, Richardson SE and Bitnun A: Neurological complications of PCR-Proven M. pneumoniae infections in children: Prodromal Illness duration may reflect pathogenetic mechanism. Clin Infect Dis 61: 1092-1098, 2015.

16. Tsiodras S,Kelesidis I,Kelesidis T,StamboulisE and Giamarellou H: Central nervous system manifestations of Mycoplasma pneumoniae infections. J Infect 51: 343-354, 2005.

17. Shibuya H, Osamura K, Hara K and Hisada T: Clinically mild encephalitis/encephalopathy with a reversible splenial lesion due to Mycoplasma pneumoniae infection. Intern Med 51: 1647-1648, 2012.

18. Sugeno N, Kawaguchi N, Hasegawa T, Kuroda T, Nakashima I, Kanbayashi T, Kusunoki S and Aoki M: A case with anti-galactocerebroside antibody-positive Mycoplasma pneumoniae meningoencephalitis presenting secondary hypersomnia. Neurol Sci 33: 1473-1476, 2012.

19. Bitnun A, Ford-Jones EL, Petric M, MacGregor D, Heurter H Nelson S, Johnson G and Richardson S: Acute childhood encephalitis and Mycoplasma pneumoniae. Clin Infect Dis 32: $1674-1684,2001$
20. Socan M, Ravnik I, Bencina D, Dove P, Zalotnik B and Jazbec J: Neurological symptoms in patients whose cerebrospinal fluid is culture- and/or polymerase chain reaction-positive for Mycoplasma pneumoniae. Clin Infect Dis 32: E31-E35, 2001.

21. Lanczik O, Lecei O, Schwarz S and Hennerici M: Mycoplasma pneumoniae infection as a treatable cause of brainstem encephalitis. Arch Neurol 60: 1813-1814, 2003.

22. Kizilkilic O and Karaca S: Influenza-associated encephalitis-encephalopathy with a reversible lesion in the splenium of the corpus callosum: Case report and literature review. AJNR Am J Neuroradiol 25: 1863-1864, 2004

23. TadaH,TakanashiJ,Barkovich AJ,ObaH,Maeda M,TsukaharaH, Suzuki M, Yamamoto T, Shimono T, Ichiyama T, et al: Clinically mild encephalitis/encephalopathy with a reversible splenial lesion. Neurology 63: 1854-1858, 2004.

24. Yamashita S, Kawakita K, Hosomi N, Naya T, Ohkita H, Kuroda $\mathrm{Y}$ and Tamiya T: Reversible magnetic resonance imaging changes associated with hypoglycemia. Case report. Neurol Med Chir (Tokyo) 50: 651-654, 2010.

25. Nishimura M, Saida T, Kuroki S, Kawabata T, Obayashi H, Saida K and Uchiyama T: Post-infectious encephalitis with anti-galactocerebroside antibody subsequent to Mycoplasma pneumoniae infection. J Neurol Sci 140: 91-95, 1996.

26. Koskiniemi M: CNS manifestations associated with Mycoplasma pneumoniae infections: Summary of cases at the University of Helsinki and review. Clin Infect Dis 17 (Suppl 1): S52-S57, 1993.

27. Candler PM and Dale RC: Three cases of central nervous system complications associated with Mycoplasma pneumoniae. Pediatr Neurol 31: 133-138, 2004

28. Daxboeck F, Blacky A, Seidl R, Krause R and Assadian O Diagnosis, treatment, and prognosis of Mycoplasma pneumoniae childhood encephalitis: systematic review of 58 cases. J Child Neurol 19: 865-871, 2004.

29. Sakoulas G: Brainstem and striatal encephalitis complicating Mycoplasma pneumoniae pneumonia: Possible benefit of intravenous immunoglobulin. Pediatr Infect Dis J 20: 543-545, 2001.

30. Guleria R, Nisar N, Chawla TC and Biswas NR: Mycoplasma pneumoniae and central nervous system complications: A review. J Lab Clin Med 146: 55-63, 2005.

31. Gucuyener K, Simsek F, Yilmaz O and Serdaroğlu A: Methyl-prednisolone in neurologic complications of Mycoplasma pneumonia. Indian J Pediatr 67: 467-469, 2000. 